\title{
Further Adaptations and Reflections by an Assertive Community Treatment Team to Serve Clients with Severe Mental Illness during COVID-19
}

\author{
Samuel Law ${ }^{1,2} \mathbb{D} \cdot$ Iline Guan ${ }^{2} \cdot$ Michaela Beder $^{1,2} \cdot$ Madeleine Ritts $^{1} \cdot$ Saadia Sediqzadah $^{1,2} \cdot$ Matthew Levy $^{1,2} \cdot$ \\ Nicole Kirwan ${ }^{1}$
}

Received: 18 May 2021 / Accepted: 26 May 2021 / Published online: 9 June 2021

(c) The Author(s), under exclusive licence to Springer Science+Business Media, LLC, part of Springer Nature 2021

We appreciate the authors' ongoing important community mental health work during the pandemic, and thank the authors for citing our original paper (Guan et al., 2021) for its conceptual framework. We applaud this timely follow up article that shares their ACT experience in Minnesota, with updated literature, reflections on the common threads between our two teams, and further suggestions and recommendations for the field.

We would like to take this opportunity, in our invited response, to highlight a number of challenges that were raised in the authors' report. To begin, the comment that the authors' ACT team was conducting business "however they could manage" is quite telling and descriptive of the issues that we were originally trying to address, namely a lack of clear guidelines and previous experiences that could prepare us for the scale, and in retrospect, the duration and

Samuel Law

Samuel.Law@Unityhealth.to

Iline Guan

iline.guan@mail.utoronto.ca

Michaela Beder

Michaela.Beder@unityhealth.to

Madeleine Ritts

Madeleine.Ritts@unityhealth.to

Saadia Sediqzadah

saadia.sediqzadah@unityhealth.to

Matthew Levy

Matthew.Levy@unityhealth.to

Nicole Kirwan

Nicole.Kirwan@unityhealth.to

1 Li Ka Shing Knowledge Institute, St. Michael's Hospital, Cardinal Carter Wing \#17029, 30 Bond Street, Toronto, ON M5B 1W8, Canada

2 Department of Psychiatry, University of Toronto, Toronto, ON, Canada impact of the Covid-19 pandemic. This shared "lost at sea" feeling has been instrumental in propelling both of our teams to engage in documenting and reflecting on how to improve on this challenge. We would invite more clinicians to join in this opportunity to learn through the current adversity.

Our initial concerns and anticipated longer-term problems related to clients struggling to cope with stress stemming from the pandemic have been observed and confirmed by the authors. The high risk of perceived threat and higher level of anxiety and depressive symptoms incurred by the pandemic as highlighted by the authors and their literature review confirm the importance of continuing the essential services provided through ACT. As well, it continues to confirm and renew our sense of professional purpose in serving our vulnerable clients.

We also find it very positive to see that good adaptive practices from the Minnesota team have informed other organizations and services to feel more confident and likely to step up in their services. We have seen similar cross-pollination of confidence inspiring adaptive new approaches across our community and ambulatory services. Our regional and provincial ACT Associations have played a strong role during the pandemic crisis in surveying and disseminating key suggestions and guidelines based on expert opinions and various teams' feedback. Having a regular ACT leadership forum to simply commiserate, normalize the stress felt by individual teams, share problems and brainstorm solutions have been invaluable. It has also served as a platform to launch new advocacy initiatives, for example, a subcommittee for homeless problems faced by ACT clients during Covid-19. Furthermore, the provincial ACT Association also added virtual learning and conferences to help the teams with their practices, including topics such as CBT for psychosis, mental health legislation and Covid-19 safety discussions. This has highlighted the importance of having a healthy, collegial, and adequately 
funded ACT organizational network, especially at a time when ACT team numbers and infrastructure are dwindling (Spivak et al., 2019).

It is reassuring to learn that the Covid-19 infection rate in the authors' team has been minimal. Similarly, the Covid-19 infection rate at the FOCUS team continues to be relatively low. For those who became infected, we found the common risk factors tended to include homelessness or high congregate housing (shelters or boarding homes), frequent needs to use of public transit, significant substance use and neurocognitive features affecting insight and judgment and ability to follow Covid-19 precautions including physical distancing, hand washing, etc. Related to on-going infection management, we have focused on close contact, with proper consent, and collaboration with public health services, designated Covid-19 shelters, and occasionally emergency rooms and in-patient stays for assessment and sheltering to protect the few Covid-19 positive patients and the community at large when the patients were too disorganized and unable to follow isolation guidelines. Since the publication of our original paper, we have had one Covid-19 related death in a person with pre-morbid health conditions and residential home outbreak, prior to any available vaccinations. Overall, the pandemic has brought home even more the reality that most of our clients live in deep poverty, due to inadequate government financial assistance rates, and many experience homelessness within the context of an insufficient stock of subsidized housing. This highlights the ongoing need for systemic level advocacy to improve income and increase housing opportunities, and ensure sufficient community and hospital supports during and post-pandemic.

The authors pointed out some heterogeneous observations in that some patients with SMI have faced the acute pandemic "like people without mental illness". One year into the pandemic, our observation is also unequivocal that many patients have shown resilience and the ability to adapt to and appreciate the necessary changes made by the team, such as having fewer in-person visits and much reduced social activities. Most have managed well with the sacrifices they had to make. We are inspired by this prevalent observation and are reminded that our medical model often primarily sees deficits and dysfunctions, and that these attitudes are often unconscious and can give rise to an overly paternalistic model of care. These successes are also a testament that a patient-focused, recovery-based form of care would result in much better service orientation and a collaborative spirit, seeing clients as capable agents in their own adaptability and recovery journey.

We also find the citing of literature from a different culture, in this case Italy, is of interest. In our team's highly diverse setting, we have found some informal impression that some factors relating to cultural and ethnic backgrounds can play a factor in people's coping. While it would be difficult to generalize, we want to promote more cultural and ethno-racial considerations in a world that is inherently inequitable (McKnight-Eily et al., 2021).

The authors also rightfully point out the concern that public health messaging and media may have neglected caregivers of SMI as an at-risk population. This applies to families and informal caregiving networks. Relatedly, there is also more extensive reporting about health care staff burn out (Jalili et al., 2021). We highly agree with the authors about the importance of ongoing attention on both of these issues. Physical burnout, emotional exhaustion and moral injury are even more heightened as the pandemic has persisted much longer than most have anticipated. Since our paper, we have initiated a "wellness Wednesdays" group for staff with restorative social activities that afforded opportunities to get to know their colleagues better and to decompress. Still, there are limitations. For instance, some staff feel too stressed to take time away from work to participate. Others point out how their wellness would be best addressed by improvements to their contracts (such as paid sick leave and vacation for those on temporary contracts.)

We share the authors wish to focus on the post "lockdown"- presumably referring to the profound social isolation related to the pandemic-mental health effects, acknowledging substance use, PTSD, anxiety and ongoing fear of contagion as well as a host of other immediate and long-term effects that can arise from living with uncertainty and distrust of some authorities. Prioritizing strategies to best prepare and update ourselves so we can better serve clients and families remains an ongoing challenge.

We agree that the incorporation of telehealth services can be one of the main innovations gained from this pandemic. The history of ACT using telemedicine technology is reaffirmed through this pandemic (Swanson \& Trestman, 2018). Our concerns do go back to a more fundamental issue of access, beyond the authors' concern about difficulty navigating the technology. Our challenge has been in securing and maintaining the technology in the first place. A good proportion of our patients have no phones, let alone internet access, or cannot maintain a data plan. When phones do become available, they are often lost or sold by patients. As the authors also noted, there are benefits of in-person contact. We want to highlight that the larger shift towards telemedicine also requires a foundation of person-to-person connection that builds towards a trusting therapeutic relationship - in a way, a relationship that has to be earned. We want to emphasize this potential caveat while we continue to make the adaptation towards increasing telemedicine services.

Furthermore, the authors helpfully point out that for some patients with SMI, the pandemic has normalized some of their pre-pandemic baseline behaviors, and there is financial aid that became more available for most clients. Of course, 
as the authors have also pointed out, there is other research that shows serious current and potential long-term effects from the pandemic. We note that the quoted study which found patients having high resilience was a study of rural patient with serious mental illness. We wonder if this is a case of studying those who did not have much services to begin with-the "have nots", who may have fared admirably and relatively better during the pandemic. This may need to be understood with finer granularity when compared to those who had lost much due to the pandemic. Ultimately, we are learning about counter intuitive findings and making pleasant new discoveries of our clients' resilience. We have also seen such in our setting and we are again advocating for understanding new practices through the lens of the journey of recovery, with principles of empowerment, collaboration, enhancing responsibility and meaning, and building identity.

The findings from the authors' informal survey of the biggest challenges that ACT team staff faced during a pandemic and of the changes in ACT work during the pandemic presents valuable information. These are likely shared by colleagues across the continent and beyond. Our team is also doing similar further research, after securing a hospital health network-based grant to study the 70 ACT teams across the province of Ontario, Canada using an onlinebased survey tool, and to convene an expert group using a Delphi approach to learn about these challenges, adaptations, and experiences. We believe that such research will further enhance our understanding and preparedness for current and future work. In particular, learning about which services are essential for an ACT team, as the authors have queried, is an important task.

As time progresses, although this was not part of the authors' report, many ACT teams are likely by now working on their clients' vaccination promotion (Warren et al., 2021). Part of our recent work has been focused on vaccine education, clarifying the consent process for those with treatment decision challenges, and assisting and mobilizing clients to procure appointments and facilitate their receiving vaccinations. We have had some success in planning mobile and on-site vaccinations for clients. The principles of community psychiatry again greatly facilitate this adaptive new focus.
Acknowledgements The authors declare no financial support for the writing of this article, and wish to acknowledge the FOCUS team members and patients for the collective effort in coping with Covid-19.

\section{Declarations}

Conflict of Interest The authors declare no potential conflicts of interest and the production of this article did not involve active human participation in research and informed consent was not applicable.

\section{References}

Guan, I., Kirwan, N., Beder, M., Levy, M., \& Law, S. (2021). Adaptations and innovations to minimize service disruptions for patients with severe mental illness during COVID-19: Perspectives and reflections from an assertive community psychiatry program. Community Mental Health Journal, 57(1), 10-17. https://doi.org/ 10.1007/s10597-020-00710-8

Jalili, M., Niroomand, M., Hadavand, F., Zeinali, K., \& Fotouhi, A. (2021). Burnout among healthcare professionals during COVID19 pandemic: A cross-sectional study. International Archives of Occupational and Environmental Health, 17, 1-8.

McKnight-Eily, L. R., Okoro, C. A., Strine, T. W., Verlenden, J., Hollis, N. D., Njai, R., Mitchell, E. W., Board, A., Puddy, R., \& Thomas, C. (2021). Racial and ethnic disparities in the prevalence of stress and worry, mental health conditions, and increased substance use among adults during the COVID-19 pandemic-United States, April and May 2020. Morbidity and Mortality Weekly Report, $70(5), 162$

Spivak, S., Cullen, B. A., Green, C., Firth, T., Sater, H., \& Mojtabai, R. (2019). Availability of assertive community treatment in the United States: 2010 to 2016. Psychiatric Services, 70(10), 948951. https://doi.org/10.1176/appi.ps.201900032

Swanson, C. L., \& Trestman, R. L. (2018). Rural assertive community treatment and telepsychiatry. Journal of Psychiatric Practice, 24(4), 269-273.

Warren, N., Kisely, S., \& Siskind, D. (2021). Maximizing the uptake of a COVID-19 vaccine in people with severe mental illness: A public health priority. JAMA Psychiatry, 78(6), 589-590.

Publisher's Note Springer Nature remains neutral with regard to jurisdictional claims in published maps and institutional affiliations. 